Research Article

\title{
Comparative study of quality of life in breast cancer patients receiving two different chemotherapy regimens using European Organization for Research and Treatment of Cancer Quality of Questionnaire-Core 30 questionnaire module; for tolerability and safety
}

\author{
Naveen Chauhan ${ }^{1}$, Kiran Haridas ${ }^{1 *}$, Indrani Poddar ${ }^{1}$, Krishna Prasad ${ }^{2}$, M. C. Alwar ${ }^{1}$, \\ Dinesh Pai Kasturi ${ }^{3}$
}

\begin{abstract}
${ }^{1}$ Department of Pharmacology, Kasturba Medical College, Mangalore, Karnataka, India, ${ }^{2}$ Department of Medical Oncology, Kasturba Medical College, Mangalore, Karnataka, India, ${ }^{3}$ Department of Oncology and Radiotherapy, Kasturba Medical College, Mangalore, Karnataka, India

Received: 09 March 2015

Revised: 17 March 2015

Accepted: 24 April 2015

*Correspondence to:

Dr. Kiran Haridas,

Email: drkiranharidas@gmail. com

Copyright: (C) the author(s), publisher and licensee Medip Academy. This is an openaccess article distributed under the terms of the Creative Commons Attribution NonCommercial License, which permits unrestricted noncommercial use, distribution, and reproduction in any medium, provided the original work is properly cited.
\end{abstract}

\begin{abstract}
Background: Breast cancer is one of the most frequent occurring cancers in women and burgeoning worldwide. It is the second most common malignancy in India after carcinoma of the uterine cervix. In clinical trials, quality of life (QOL) outcome measurements is an important as endpoints with improving subjects physical, emotional, and social well-being.

Methods: In this study, we were evaluated the comparison of the QOL in breast cancer patients on anthracycline-based regimen (six cycles of 5-fluorouracil, adriamycin, and cyclophosphamide [FAC] for a period of 18 weeks) and taxane-containing regimen (four cycles of adriamycin and cyclophosphamide [AC] followed by four cycles of paclitaxel [PTX] for a period of 24 weeks) using European Organization for Research and Treatment of Cancer Quality of Questionnaire-Core 30.

Results: During first 3 months of therapy, both treatment groups exhibited a reduction in health-related QOL (HRQOL) with no clinically significant difference between them. The effect on HRQOL was less evident 3 weeks after completing chemotherapy with HRQOL of both groups returning to near baseline scores.

Conclusions: Both treatment regimens (FAC and AC $\rightarrow$ PTX [AC followed by PTX]) were equally tolerated in patients.
\end{abstract}

Keywords: Breast cancer, Health-related quality of life, 5-Fluorouracil, Adriamycin, Cyclophosphamide, Paclitaxel

\section{INTRODUCTION}

Quality of life (QOL) outcome measurements have become increasingly important as endpoints in clinical trials. More and more emphasis is being placed not only on treating the patients but also on improving their physical, emotional, and social well-being. The term "QOL" covers the entire gamut of an individual's feelings, beliefs, and perceptions of life. In medicine, QOL takes a more restricted view, because the focus is primarily on those aspects of QOL that are affected by the disease and its treatment. This view is more appropriately labeled health-related QOL (HRQOL). Most agree that HRQOL is a multidimensional construct. ${ }^{1}$ Breast cancer is a malignant proliferation of epithelial cells lining the ducts or lobules of the breast. Breast cancer is one of the most common cancers in women worldwide, with an incidence of about 570,000 patients per year. ${ }^{2}$ Carcinoma of the breast is the leading cause of cancer in women all 
over the world and the second most common malignancy in India after carcinoma of the uterine cervix. ${ }^{3}$ The probability of developing breast cancer increases throughout life. The mean and the median age of women with breast cancer are between 60 and 61 years. Most breast cancers arise from the intermediate ducts and are invasive ${ }^{4}$ (invasive ductal, infiltrating ductal). Stage of breast cancer is the most reliable indicator of prognosis. When cancer is localized to the breast, with no evidence of regional spread, the clinical cure rate with most accepted methods of therapy is $75 \%$ to $>90 \%$. When the axillary lymph nodes are involved with tumor, the survival rate drops to $50-70 \%$ at 5 years and probably around $25-40 \%$ at 10 years. ${ }^{4}$ In general, breast cancer appears to be somewhat more malignant in younger than in older women. ${ }^{4}$ Following surgery and possible radiation therapy, systemic therapy improves survival and is advocated for most patients with curable breast cancer. The objective of adjuvant systemic therapy is to eliminate the occult metastases in distant organs while they are microscopic and most vulnerable. ${ }^{4}$

The World Health Organization defined health as "a state of complete physical, mental, and social well-being, and not merely the absence of disease." HRQOL may incorporate a wide variety of issues including general health, physical symptoms, functionality, toxicity, emotional well-being, cognitive issues, role functioning, social well-being, sexual functioning, existential/spiritual issues, financial status, job satisfaction, and living conditions. The most appropriate way to measure an individual's QOL is self-reporting directly by the patient. QOL ratings by physicians or other medical personnel have been found to differ significantly from patient reports. ${ }^{1,5}$

QOL studies have allowed oncologists to understand better the impact of various forms of cancer therapy on the patient's well-being and functioning and have guided caretakers in making recommendations to improve the patient's overall QOL. Knowledge gained from a QOL study can be used to communicate accurately to future patients treatment-related morbidities or resultant limitations in the functional and emotional well-being that are likely to occur. One of the most interesting aspects of baseline QOL is its ability to serve as an independent prognostic factor for survival or locoregional control. ${ }^{6}$

On the basis of the superiority of anthracycline-containing regimens in metastatic breast cancer, both doxorubicin and epirubicin have been studied extensively in the adjuvant setting. The use of six cycles of 5-fluorouracil, adriamycin and cyclophosphamide (FAC) or 5-fluorouracil, epirubicin and cyclophosphamide (FEC) has shown improved survival compared with cyclophosphamide, methotrexate, and 5-fluorouracil (CMF) alone. For node-positive patients and recently selected node-negative patients, taxanes are frequently combined with anthracycline-based regimens. Initial studies demonstrated a $20 \%$ proportional reduction in recurrence and a $4 \%$ absolute improvement in disease- free survival with the addition of paclitaxel (PTX) to an adriamycin and cyclophosphamide (AC) regimen. PTX is Food and Drug Administration - approved for and increasingly used as adjuvant therapy in node-positive breast cancer. The overall duration of adjuvant chemotherapy still remains uncertain. However, based on the meta-analysis performed in the Oxford overview (early breast cancer trialists' collaborative group), the current recommendation is for 3-6 months of the commonly used regimens. The addition of taxanes required an additional duration of therapy of up to 6 months. $^{4}$

The present study focused on the comparison of the QOL in patients on anthracycline-based regimen (six cycles of FAC for a period of 18 weeks) and taxane-containing regimen (four cycles of AC followed by four cycles of PTX for a period of 24 weeks) using European Organization for Research and Treatment of Cancer Quality of QuestionnaireCore 30 (EORTC-C30, version 3) and also for safety and tolerability.

\section{METHODS}

This was an open, randomized, prospective study approved by Institutional Ethics Committee. The present study was performed in the Department of Oncology, KMC Hospital in Attavar during the period of May 2007 to May 2008.

The primary objective was to compare the QOL in patients who are on anthracycline-based regimen (six cycles of FAC) and taxane-containing regimen (four cycles of AC followed by four cycles of PTX). The secondary outcome was to compare safety and tolerability between the anthracyclinebased (six cycles of FAC) and taxane-containing regimen (four cycles of AC followed by four cycles of PTX).

\section{Study participants}

All patients between 18 and 70 years of age with women with any stage of carcinoma of breast, using American Joint Committee on Cancer Staging Manual, 6th edition. Springer, 2002 provided written informed consent prior to the beginning of study procedures. The prior hormonal therapy is allowed in either the metastatic or adjuvant setting, but must be discontinued prior to the first study drug administration. Eastern Cooperative Oncology Group performance status $0-1$ and an anticipated life expectancy of $\geq 6$ months. Patients with adequate bone marrow function (absolute neutrophil count $>4000 / \mathrm{mm}^{3}$, platelet count $>100,000 / \mathrm{mm}^{3}$, and hemoglobin $>10 \mathrm{~g} / \mathrm{dL}$ ), adequate liver and kidney function [total bilirubin within normal limits, aspartate transaminase $<3 \times$ upper limit of normal (ULN) (or $\leq 5 \times$ ULN in the presence of metastatic liver disease) alanine aminotransferase (ALT) $<3 \times$ ULN (or $\leq 5 \times$ ULN in the presence of metastatic liver disease) and serum creatinine $<1.5 \mathrm{mg} / \mathrm{dL}$ ], adequate cardiac functions, negative serum or urine $\beta$-human chorionic gonadotropin pregnancy test 
for women of childbearing potential, adequate birth control measures by women of childbearing potential to prevent pregnancy during the study.

This study included 20 patients with breast cancer who had undergone surgery (modified radical mastectomy) and were randomized into two groups. First group received anthracyclinebased regimen FAC $\left(600 \mathrm{mg} / \mathrm{m}^{2}\right.$ cyclophosphamide, $50 \mathrm{mg} / \mathrm{m}^{2}$ adriamycin, and $600 \mathrm{mg} / \mathrm{m}^{2}$ 5-fluorouracil) every 3 weeks for six cycles. Second group received taxanes-based regimen AC (600 mg/m² cyclophosphamide and $60 \mathrm{mg} / \mathrm{m}^{2}$ adriamycin) every 3 weeks for four cycles followed by $175 \mathrm{mg} / \mathrm{m}^{2}$ PTX given as a 3 hrs every 3 weeks for four cycles. ${ }^{7-9}$

Before assignment, patients were required to undergo history and physical examination (including gynecologic examination for patients with an intact uterus and/or ovaries), hematologic studies and chemistries, chest X-ray (within 3 months of random assignment), and ECG and bilateral mammogram (within 1 year from random assignment). Patients were also required to have history and physical examination along with hematologic studies and chemistries on day 1 and every 3 weeks before each cycle of the chemotherapy regimen.

Tolerability addresses the side effects of drugs that relate specifically to the perception of patients. Laboratory investigations including hemoglobin, total leukocyte count, platelet count, random blood sugar, alanine aminotransferase, total bilirubin, blood urea, and serum creatinine were mandatory on days 20-21 of each cycle. Clinical toxicity was carefully evaluated on day 21 of each cycle.

Premedication with dexamethasone $8 \mathrm{mg}$ intravenous (IV), ondansetron $8 \mathrm{mg}$ or granisetron $3 \mathrm{mg}$ IV, ranitidine $50 \mathrm{mg}$ IV was given prior to the administration of cyclophosphamide, adriamycin, and 5-fluorouracil in each chemotherapy cycle.

Antibiotics such as cefotaxime $1 \mathrm{~g}$ IV, ciprofloxacin 200-500 mg IV, azithromycin $500 \mathrm{mg}$ oral tablets were used to combat infections.

Before each cycle of PTX, patients were required to receive premedication with dexamethasone $20 \mathrm{mg}$ PO (12 and $6 \mathrm{hrs}$ before PTX infusion), diphenhydramine $50 \mathrm{mg}$ IV (1 hr before PTX infusion), ranitidine $50 \mathrm{mg}$ IV (1 hr before PTX infusion), and alprazolam $0.25 \mathrm{mg}$. Other medications prescribed were: paracetamol $500 \mathrm{mg}$ tablet, bisacodyl $5 \mathrm{mg}$ tablet, becosules capsule, lorazepam $1 \mathrm{mg}$ tablet, and domperidone $10 \mathrm{mg}$.

EORTC-C30 questionnaire module is available in three languages; English, Kannada, and Malayalam. The actual method consists of the use of EORTC QLQ-C30 QOL questionnaire given to the patients which contain questions regarding their daily lives. QOL data in the form of the EORTC QLQ-C30 were collected at baseline, at each cycle of chemotherapy and once at follow-up of 3 weeks after therapy. If patients were too ill to attend the clinic, they could be contacted at home or visited in the hospital to complete the questionnaire. QLQ-C30 version 3.0 incorporates five functional scales: physical functioning $(\mathrm{PF})$, role functioning (RF), cognitive functioning $(\mathrm{CF})$, emotional functioning (EF) and social functioning (SF); three symptom scales: fatigue (FA), pain (PA), nausea and vomiting (NV); a global health status/QOL scale (QL), and six single items assessing additional symptoms: dyspnea (DY), loss of appetite (AP), insomnia (SL), constipation (CO), diarrhea (DI), and a single item on the perceived financial impact of the disease (FI). All of the scales and single-item measures range in score from 0 to 100. A high scale score represents a higher response level. Thus, a high score for a functional scale represents a high/healthy level of functioning; a high score for the global health status/ QOL represents a high QOL; but a high score for a symptom scale/item represents a high level of symptomatology/ problems.

\section{Statistical analysis}

Sample size was based on the primary endpoint. Data for the EORTC QLQ-C30 were scored using the EORTC Scoring Manual. ${ }^{10}$ Comparisons were made of the primary end points (HRQOL) of both the treatment groups for all patients included in the study. Secondary end points were tolerability and safety of the two separate regimens. MannWhitney U-test was applied for comparison between two groups (FAC and AC $\rightarrow$ PTX regimens). Student's t-test was applied for parameters such as age (years), weight (kg), height $(\mathrm{cm})$, systolic blood pressure $(\mathrm{mmHg})$, and diastolic blood pressure $(\mathrm{mmHg})$. Chi-square test $\left(\chi^{2}\right)$ was applied for parameters including menstrual history, number of positive nodes, age at surgery, clinical tumor size, histologic type, clinical T-stage, tumor grade, and operation (modified radical mastectomy). The level of significance was set at $\mathrm{p}=0.05$.

\section{RESULTS}

Consolidated Standards of Reporting Trials diagram of patient disposition is depicted in Figure 1. A total number of 28 patients were screened, of which 10 patients in each anthracycline-based regimen (six cycles of FAC) and taxanes-based regimen (four cycles of AC followed by four cycles of PTX) groups completed the study in 18 weeks and 24 weeks, respectively. Four patients were screened failure and four patients did not complete the study due to lost to follow-up, development of infection, and changed to different chemotherapy regimen.

\section{Patients' characteristics at baseline}

The patient's characteristics at baseline were recorded during visit 1 and are tabulated in Table 1. There were no statistically 


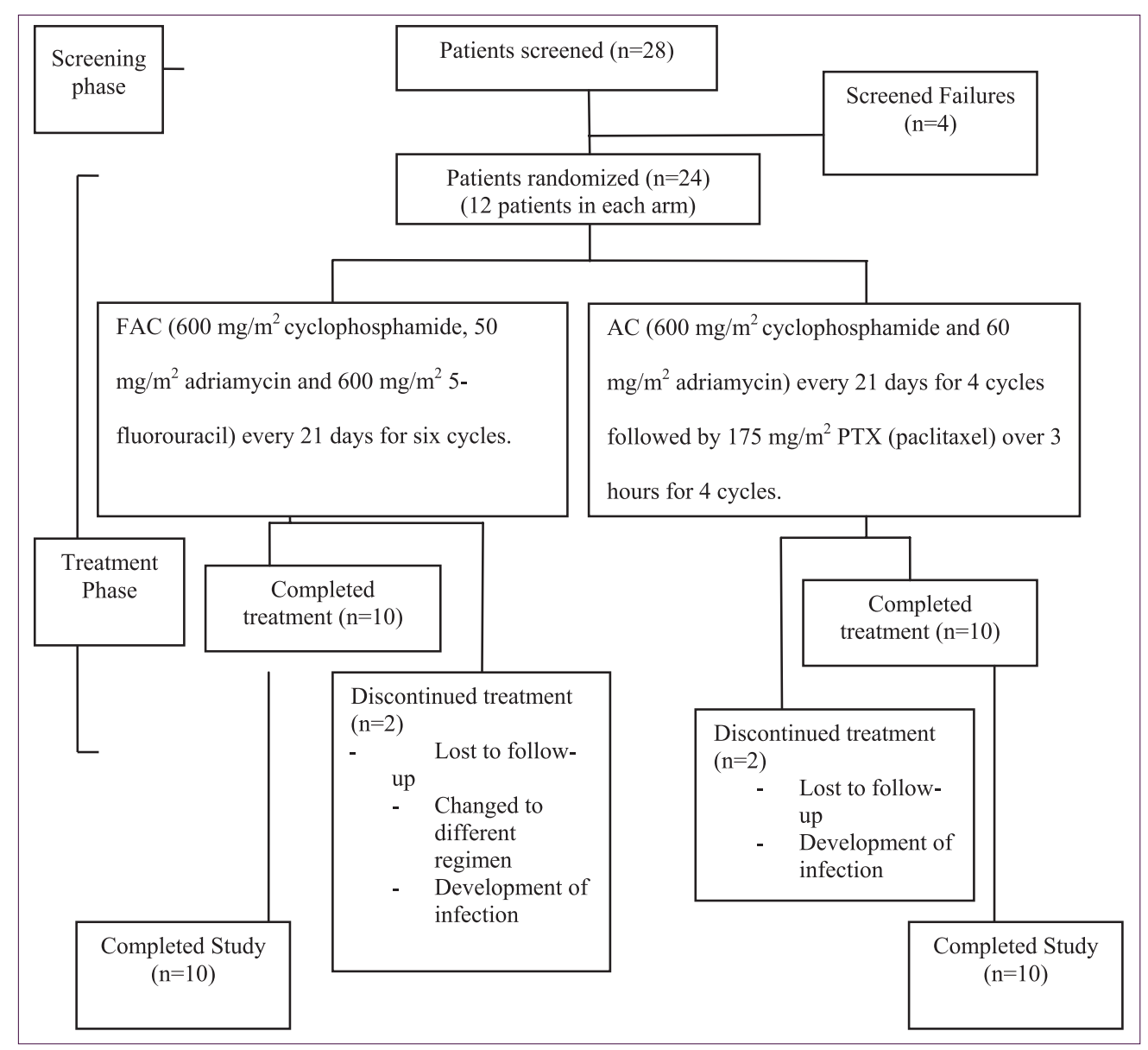

Figure 1: Consolidated Standards of Reporting Trials diagram of patient disposition.

significant differences between treatment groups on any baseline demographic measures.

As shown in Table 1, about half of the patients were younger than 50 years and 5\% were 60 years of age or older. About $65 \%$ of women had attained menopause. Approximately $60 \%$ of the patients in FAC arm and $70 \%$ in $\mathrm{AC} \rightarrow$ PTX arm had tumors $>4 \mathrm{~cm}$ in maximum diameter.

$70 \%$ of the patients in FAC arm and $40 \%$ in $\mathrm{AC} \rightarrow \mathrm{PTX}$ arm had one to three positive nodes on pathologic examination, and only $20 \%$ of the patients had $>4$ positive nodes.

All patients had the infiltrating ductal type of breast cancer and undergone modified radical mastectomy prior to the administration of chemotherapy.

$90 \%$ of patients had clinical tumor Stage T2 and T3, and only $10 \%$ had T4 clinical tumor stage. $85 \%$ of patients had tumor stage of intermediate type.

In general, any chemotherapy treatment deteriorated all HRQOL domains during the treatment period (Table 2).

Patients assigned to six cycles of FAC treatment regimen did not show statistically significant difference $(p>0.05)$ in comparison to patients on four cycles of AC $\rightarrow$ four cycles of
PTX (PTX) in global health status/QOL scale and functional scales including PF, RF, and CF (Figure 2).

There is a statistically significant difference between the two groups in EF $(p=0.05)$ and SF $(p=0.029)$ at 15 th week of therapy (Figure 2).

Results from symptom scales/items of HRQOL including pain, dyspnea, nausea and vomiting (NV), insomnia, constipation, diarrhea, and financial difficulties did not show statistically significant difference $(p>0.05)$ between two groups. However, there is a statistically significant difference between the two groups in fatigue at 15th week (significantly worse in $4 \mathrm{AC} \rightarrow 4$ PTX treatment group [p=0.045]; [Figure 2]) and appetite loss at 9th, 12th, and 18 th week of therapy (significantly worse in 4 AC $\rightarrow 4$ PTX treatment group with highly significant at 9th week $[\mathrm{p}=0.002]$ and significant at 12 th $[\mathrm{p}=0.029]$ and 18 th week $(\mathrm{p}=0.019) ;$ [Figure 2]).

Severe anemia was defined as a hemoglobin concentration of $10 \mathrm{~g} / \mathrm{dl}$ or less after a cycle of chemotherapy. Anemia was observed in one patient in $4 \mathrm{AC} \rightarrow 4$ PTX treatment group and in three patients in FAC treatment group at 3rd week; two patients in $4 \mathrm{AC} \rightarrow 4$ PTX treatment group, and in one patient in FAC treatment group at 6th week; one patient in FAC treatment group at 9th week and in one patient in FAC 
Table 1: Patient's characteristics at baseline.

\begin{tabular}{|c|c|c|}
\hline Demographic parameters & FAC $(n=10)$ & $4 \mathrm{AC} \rightarrow 4$ PTX $(\mathrm{n}=10)$ \\
\hline Age (years), mean (SD) & $51(7.73)$ & $45.2(7.91)$ \\
\hline Weight (kg), mean (SD) & $51.7(4.99)$ & $60.1(7.72)$ \\
\hline Height $(\mathrm{cm})$, mean $(\mathrm{SD})$ & $159.1(4.14)$ & $160.4(3.98)$ \\
\hline Systolic blood pressure (mmHg) mean (SD) & $122.2(8.81)$ & $120.4(7.3)$ \\
\hline Diastolic blood pressure (mmHg), mean (SD) & $80(5.34)$ & $77.2(5.0)$ \\
\hline \multicolumn{3}{|l|}{ Menstrual history (n [\%]) } \\
\hline Premenopausal & $4(40)$ & $3(30)$ \\
\hline Postmenopausal & $6(60)$ & $7(70)$ \\
\hline \multicolumn{3}{|l|}{ Number of positive (n [\%]) } \\
\hline 0 & $3(30)$ & $4(40)$ \\
\hline $1-3$ & $7(70)$ & $4(40)$ \\
\hline $4-9$ & $0(0)$ & $2(20)$ \\
\hline \multicolumn{3}{|l|}{ Age at surgery, years (n [\%]) } \\
\hline$<39$ & $1(10)$ & $2(20)$ \\
\hline $40-49$ & $4(40)$ & $3(30)$ \\
\hline $50-59$ & $4(40)$ & $5(50)$ \\
\hline$>60$ & $1(10)$ & $0(0)$ \\
\hline \multicolumn{3}{|l|}{ Clinical tumor size, $\mathrm{cm}(\mathrm{n}[\%])$} \\
\hline $2.1-4$ & $4(40)$ & $3(30)$ \\
\hline$>4.1$ & $6(60)$ & $7(70)$ \\
\hline \multicolumn{3}{|l|}{ Histologic type (n [\%]) } \\
\hline Ductal & $10(100)$ & $10(100)$ \\
\hline \multicolumn{3}{|l|}{ Clinical T-stage (n [\%]) } \\
\hline $\mathrm{T} 2$ & $6(60)$ & $3(30)$ \\
\hline $\mathrm{T} 3$ & $4(40)$ & $5(50)$ \\
\hline $\mathrm{T} 4$ & $0(0)$ & $2(20)$ \\
\hline \multicolumn{3}{|l|}{ Tumor grade (n [\%]) } \\
\hline Intermediate & $8(80)$ & $9(90)$ \\
\hline Poor & $2(20)$ & $1(10)$ \\
\hline \multicolumn{3}{|l|}{ Operation (n [\%]) } \\
\hline Mastectomy & $10(100)$ & $10(100)$ \\
\hline
\end{tabular}

SD: Standard deviation, FAC: 5-Fluorouracil, adriamycin and cyclophosphamide, PTX: Paclitaxel, AC: Adriamycin and cyclophosphamide

Table 2: Overall HRQOL by treatment group.

\begin{tabular}{|lccc|}
\hline $\begin{array}{l}\text { Treatement } \\
\text { period }\end{array}$ & \multicolumn{2}{c}{ Mean [SD] } & p \\
\cline { 2 - 4 } & $\mathbf{4} \mathbf{A C} \rightarrow$ 4 PTX & 6 FAC & \\
\hline Baseline & $46.6(11.06)$ & $45.09(11.24)$ & $0.72 \mathrm{~ns}$ \\
\hline 6 weeks & $33.43(15.55)$ & $32.63(13.21)$ & $0.97 \mathrm{~ns}$ \\
\hline 9 weeks & $28.43(10.50)$ & $27.63(8.85)$ & $0.95 \mathrm{~ns}$ \\
\hline 12 weeks & $34.23(10.77)$ & $26.73(6.60)$ & $0.08 \mathrm{~ns}$ \\
\hline 15 weeks & $35.93(7.98)$ & $27.86(12.26)$ & $0.09 \mathrm{~ns}$ \\
\hline 18 weeks & $40.90(10.61)$ & $40.93(10.67)$ & $0.84 \mathrm{~ns}$ \\
\hline 21 weeks & $40.90(9.92)$ & - & - \\
\hline 24 weeks & $46.83(13.21)$ & - & - \\
\hline
\end{tabular}

HRQOL: Health-related quality of life, PTX: Paclitaxel, SD: Standard deviation, Ns: Non-significant treatment group at 15 th week. These patients were admitted in the hospital and managed with blood transfusion support. However, there was no statistically significant difference between two groups in hemoglobin levels and the red cell transfusion requirements $(\mathrm{p}>0.05)$.

There were no statistically significant differences in platelet count, total bilirubin, random blood sugar, blood urea, and serum creatinine between the $4 \mathrm{AC} \rightarrow 4$ PTX and FAC treatment groups $(p>0.05)$. Statistically significant differences were found in the alanine aminotransferase levels at 9 th $(p=0.045)$ and 12 th week $(p=0.013)$ between the $4 \mathrm{AC} \rightarrow 4$ PTX and FAC treatment group; plasma levels of ALT (units/L) were more raised in FAC treatment group but within the upper normal limit $(\mathrm{ALT}<3 \times \mathrm{ULN})$. Two patients in $4 \mathrm{AC} \rightarrow 4$ PTX treatment group at 9 th and 15 th week, 


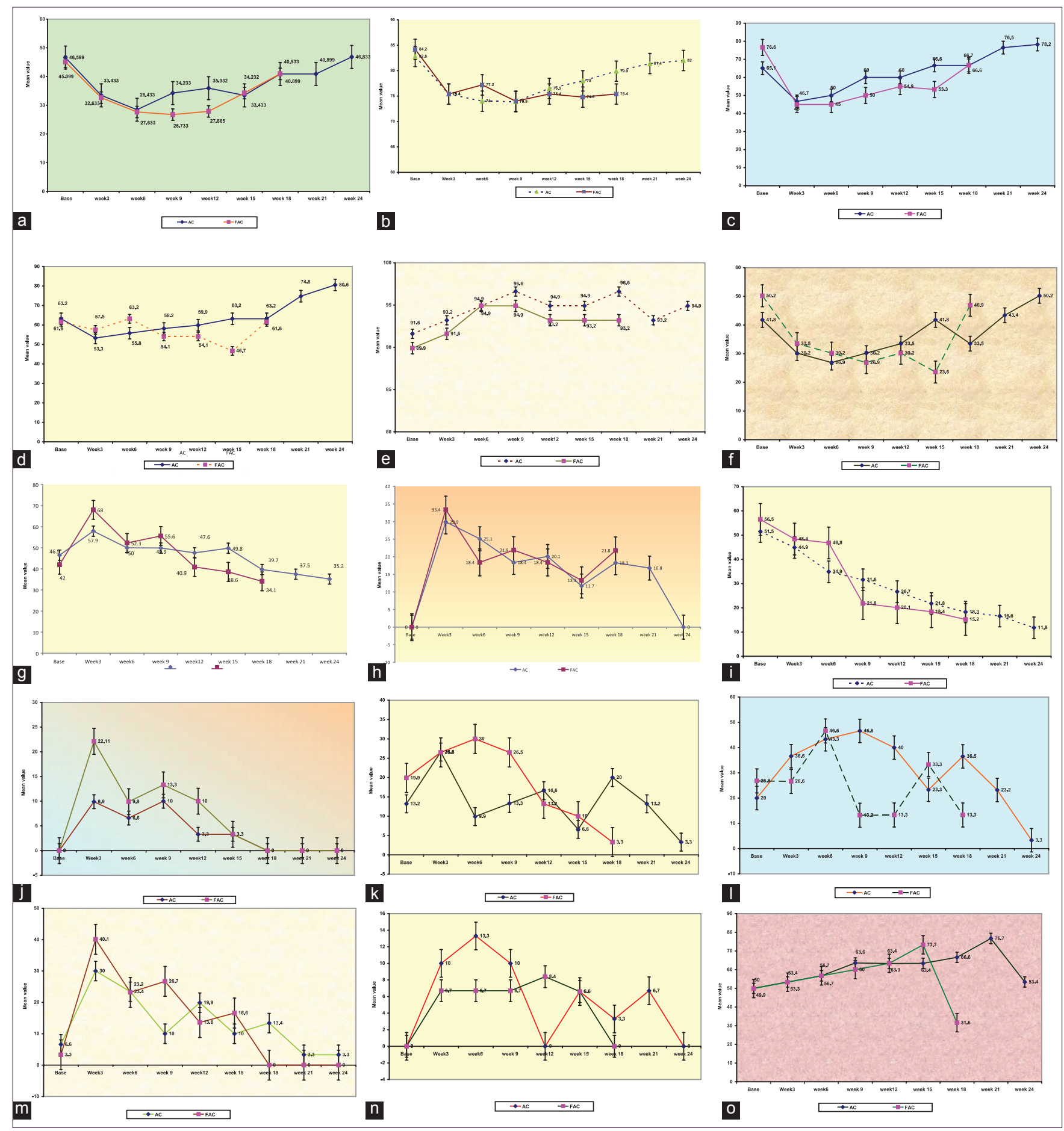

Figure 2: Individual scales of health-related quality of life (HRQOL-C-30 version 3.0), (a) Comparison of mean global health status/QOL between two groups, (b) comparison of mean physical functioning between two groups, (c) comparison of mean role functioning between two groups, (d) comparison of mean emotional functioning between two groups, (e) comparison of mean cognitive functioning between two groups, (f) comparison of mean social functioning between two groups, (g) comparison of mean fatigue between two groups, (h) comparison of mean nausea and vomiting between two groups, (i) comparison of mean pain between two groups, (j) comparison of mean dyspnea between two groups, (k) comparison of mean insomnia between two groups, (l) comparison of mean appetite loss between two groups, $(\mathrm{m})$ comparison of mean constipation between two groups, $(\mathrm{n})$ comparison of mean diarrhea between two groups, (o) comparison of mean financial difficulties between two groups.

respectively, and two patients in FAC treatment group at 6th week developed infection and were hospitalized and given antibiotics such as cefotaxime, ciprofloxacin, or azithromycin.
There was no statistically significant difference between the two groups in total leukocyte count $(p>0.05)$ except at 6 th week $(p=0.049)$ with more elevated levels in FAC group. 
Other side effect such as alopecia was observed in all patients $(100 \%)$ in both treatment groups within 1-month period of chemotherapy. Stomatitis was observed in 3 patients in FAC treatment group and 2 patients in $\mathrm{AC} \rightarrow$ PTX treatment group.

No toxic deaths (septic or non-septic) occurred in this study.

\section{DISCUSSION}

In our study, we observed that initial HRQOL after treatment (modified radical mastectomy) for breast cancer deteriorated in both FAC and AC $\rightarrow$ PTX (AC followed by PTX) groups, but did not show any clinically meaningful significant difference between two groups. These negative effects during initial stages of treatment were noted for many of the HRQOL scales. Because many women with locally advanced breast cancer have a treatment that can substantially lengthen survival, maintaining a good HRQOL is crucial. Chemotherapy can help sustain a good HRQOL over time by reducing the disease burden.

The optimal timing and frequency of QOL questionnaire administration varies among clinical trials. Factors influencing timing and frequency include the hypothesis being tested, the natural course of the disease, the treatment protocol, and its anticipated side effects. ${ }^{11}$ Studies comparing $\mathrm{AC}$ with $\mathrm{CMF}$ have shown that treatments with anthracycline-containing regimens are at least as effective, and perhaps more effective. ${ }^{11}$

The addition of a taxanes (PTX or docetaxel) to an anthracycline-based regimen appears to improve disease-free and overall survival. ${ }^{12}$

National Surgical Adjuvant Breast and Bowel Project B-28 observed that the addition of four cycles of adjuvant PTX after four cycles of adjuvant doxorubicin and cyclophosphamide (AC) resulted in significant improvement in disease-free survival, but no significant improvement in overall survival with acceptable toxicity in patients with resected operable breast cancer and histologically positive axillary nodes. ${ }^{13}$

Bottomley et al., assessed the clinical benefit of a doseintensified anthracycline-based regimen $\left(830 \mathrm{mg} / \mathrm{m}^{2}\right.$ cyclophosphamide and $120 \mathrm{mg} / \mathrm{m}^{2}$ epirubicin both given intravenously on day 1 , and $5 \mu \mathrm{g} / \mathrm{kg}$ filgrastim per day given subcutaneously on days 2-13, for six cycles every 14 days: 3 months' treatment) compared with standard treatment ( $75 \mathrm{mg} / \mathrm{m}^{2}$ cyclophosphamide given orally on days $1-14$, and $60 \mathrm{mg} / \mathrm{m}^{2}$ epirubicin and $500 \mathrm{mg} / \mathrm{m}^{2}$ fluorouracil both given intravenously on days 1 and 8 , for six cycles every 28 days: 6 months' treatment) and also showed dose-intensified treatment had a significantly lower HRQOL score during the first 3 months than did those assigned standard treatment for patients with locally advanced breast cancer. However, scores returned to near baseline, with no difference between groups at 12 months. ${ }^{14}$
The results of this systemic review support the use of taxanes as adjuvant chemotherapy for women with early breast cancer and involved lymph nodes. The strongest support is for the addition of four cycles of PTX to four cycles of AC or for the substitution of six cycles of FAC with six cycles of docetaxel, doxorubicin, and cyclophosphamide. This effect is independent of hormone-receptor status, and the evidence does not support restricting the use of taxanes to women with hormone-receptor negative tumors. ${ }^{15}$

Yvonne Brandberg et al., observed patients in the standard FEC therapy followed by marrow-supported cyclophosphamide, thiotepa, and carboplatin therapy had more pronounced deterioration of their HRQOL using the EORTC QOL Questionnaire-C30 and EORTC Breast Cancer Module-23 during the 1st year after random assignment to treatment than tailored FEC therapy for nine courses, but returned more quickly to original levels compared with patients receiving the more protracted therapy with the dose-escalated and tailored FEC regimen. Thus, although both treatments had substantial negative impact on most HRQOL areas, EF increased, and most patients seemed to recover after this burdensome adjuvant treatment. ${ }^{16}$

Phase III randomized study evaluating the toxicity and HRQOL in breast cancer patients receiving adjuvant docetaxel, doxorubicin, cyclophosphamide (TAC), or FAC showed that HRQOL of patients treated with TAC is worse than of those treated with FAC, but improves with the addition of primary prophylactic granulocyte colony-stimulating factor, particularly in the final part of chemotherapy treatment. ${ }^{17}$

In our study, patients assigned to six cycles of FAC treatment group did not show statistically significant difference $(\mathrm{p}>0.05)$ in comparison to patients on 4 AC $\rightarrow 4$ PTX (six cycles of AC followed by four cycles of PTX) regimen in global health status/QOL scale and functional scales including PF, RF, and CF except that there was statistically significant difference in EF and SF at 15th week between the two treatment groups.

Assessment of symptom scales HRQOL including pain, dyspnea, NV, insomnia, constipation, diarrhea, and financial difficulties has not shown any statistically significant difference between two groups. However, there is a statistically significant difference between the two groups in fatigue at 15 th week (significantly worse in $4 \mathrm{AC} \rightarrow 4$ PTX [four cycles of AC followed by four cycles of PTX] treatment group) and appetite loss at 9th, 12th, and 18th week (significantly worse in treatment group). The significant difference in appetite loss at the 18th week between two regimens could be explained due to completion of chemotherapy in FAC treatment group.

Anemia was more commonly seen in FAC treatment group (6 patients in FAC treatment group and 3 patients in $4 \mathrm{AC} \rightarrow 4$ PTX [four cycles of AC followed by four cycles 
of PTX] treatment group). These patients received blood transfusion support. However, there was no statistically significant difference between two groups in hemoglobin levels and the red cell transfusion requirements. Patients with chemotherapy-induced anemia presented with symptoms such as fatigue, weakness, impaired cognitive function, respiratory distress, and chest pain. They were treated with the aim of increasing hemoglobin concentration or preventing a decrease in hemoglobin concentration while continuing chemotherapy. A benefit analysis showed that an increase of hemoglobin concentration to above $10 \mathrm{~g} / \mathrm{L}$ was associated with a substantial improvement in QOL.?

Infection was observed in two patients in $4 \mathrm{AC} \rightarrow 4$ PTX (four cycles of AC followed by four cycles of PTX) group at 9th and 15th week and two patients in FAC group at 6th week for which they were hospitalized and received antibiotics such as cefotaxime, ciprofloxacin, or azithromycin. However, there was no statistically significant difference between the two groups in total leukocyte count except at 6th week with more elevated levels in FAC group. Overall, the occurrence of doselimiting toxic effects is thought to be unpredictable. Therefore, oncologists generally take action to counter these effects only after they have arisen (reactively). Such management involves rescuing the patients from an unpleasant potentially dangerous situation and adjusting the chemotherapy regimen, such as dose reduction or introduction of supportive care drugs (epoetin alfa or filgrastim) to prevent further occurrences. Clinical care could be improved substantially if toxic effects could be predicted accurately and if steps could be taken in advance to prevent them (proactively). Such care might include the use of appropriate supportive care, forewarning of the patient and initiation of an intensive early-monitoring scheme and action plan for early intervention.

Patients in two different regimens (FAC and AC $\rightarrow$ PTX groups) have not shown any clinically meaningful difference in platelet count, total bilirubin, random blood sugar, blood urea, and serum creatinine. There is statistically significant difference was found in the alanine aminotransferase levels at 9th and 12th week plasma levels of ALT [units/L] were more raised in FAC group but within the upper normal limit $($ ALT $<3 \times \mathrm{ULN})$.

During our study period, we could obtain only 20 patients who completed the study within the given time period. It could have been better if we had a large number of sample size.

No internationally validated and translated HRQOL module specific for breast cancer was available in local languages (Kannada and Malayalam) which is an unavoidable limitation of this study. Four patients dropped from the study due to the following reason. First patient in FAC treatment group did not complete the study due to the development of infection at second visit. Second patient in FAC treatment group did not complete the study because of a change in the treatment regimen. First patient in AC $\rightarrow$ PTX group treatment group was lost to follow-up. Second patient in $\mathrm{AC} \rightarrow$ PTX group treatment group did not complete the study due to the development of infection at third visit.

Unlike earlier studies, our study included patients in all stages but not with distant metastases (M0). The followup also was not done later as the patients were put on radiotherapy which requires a new HRQOL study.

Long-term follow-up as in the study conducted by Bottomley et al. could not be done in our study. In this multicenter randomized study baseline assessments of HRQOL were done before randomization; then once a month for the first 3 months; and at months $6,9,12,18,26,34,42,48$, and 54 in 224 patients with locally advanced breast cancer. HRQOL was compared between dose-intensified anthracycline-based regimen $\left(830 \mathrm{mg} / \mathrm{m}^{2}\right.$ cyclophosphamide and $120 \mathrm{mg} / \mathrm{m}^{2}$ epirubicin both given intravenously on day 1 , and $5 \mu \mathrm{g} / \mathrm{kg}$ filgrastim per day given subcutaneously on days 2-13, for six cycles every 14 days: 3 months' treatment) and standard treatment $\left(75 \mathrm{mg} / \mathrm{m}^{2}\right.$ cyclophosphamide given orally on days $1-14$, and $60 \mathrm{mg} / \mathrm{m}^{2}$ epirubicin and $500 \mathrm{mg} / \mathrm{m}^{2}$ fluorouracil both given intravenously on days 1 and 8 , for six cycles every 28 days: 6 months' treatment). ${ }^{18}$

In another HRQOL study in breast cancer patients receiving adjuvant docetaxel, doxorubicin, and cyclophosphamide (TAC) or FAC, HRQOL was analyzed at baseline, at six prospective time points corresponding to chemotherapy cycles and then at 44,68 , and 120 weeks of the study. ${ }^{19}$

\section{CONCLUSION}

Our findings show that:

1. Both FAC and AC $\rightarrow$ PTX (AC followed by PTX) treatment groups reduced HRQOL especially during the first 3 months of therapy with no clinically meaningful difference between two groups

2. The effect on HRQOL was less evident 3 weeks after completing chemotherapy, with HRQOL of both groups returning to near baseline scores

3. This study showed that patients can tolerate both treatment regimens [FAC and AC $\rightarrow$ PTX (AC followed by PTX)] equally.

Further, large numbers of breast cancer patients are needed to support the findings of this study.

Funding: No funding sources

Conflict of interest: None declared

Ethical approval: The study was approved by the Institutional

Ethics Committee

\section{REFERENCES}

1. Siddiqui F, Kachnic LA, Movsas B. Quality-of-life outcomes in oncology. Hematol Oncol Clin North Am. 2006;20(1):165-85. 
2. Parkin DM. Global cancer statistics in the year 2000. Lancet Oncol. 2001;2(9):533-43.

3. Paymaster JC. Cancer of the breast in Indian women. Surgery. 1956;40:372-7.

4. McPhee SJ, Papadakis MA, Tierney LM. Breast. In: Current Medical Diagnosis and Treatment. 47th Edition. New York, NY: McGraw-Hill; 2008.

5. Slevin ML, Plant H, Lynch D, Drinkwater J, Gregory WM. Who should measure quality of life, the doctor or the patient? Br J Cancer. 1988;57(1):109-12.

6. Aaronson NK, Ahmedzai S, Bergman B, Bullinger M, Cull A, Duez NJ, et al. The European organization for research and treatment of cancer QLQ-c30: a quality-of-life instrument for use in international clinical trials in oncology. J Natl Cancer Inst. 1993;85(5):365-76.

7. Sweetman SC. In: Martindale Complete Drug Reference. 35th Edition. London, UK: Pharmaceutical Press; 2007.

8. NowakAK, Wilcken NR, Stockler MR, Hamilton A, Ghersi D. Systematic review of taxane-containing versus non-taxanecontaining regimens for adjuvant and neoadjuvant treatment of early breast cancer. Lancet Oncol. 2004;5(6):372-80.

9. Mamounas EP, Bryant J, Lembersky B, Fehrenbacher L, Sedlacek SM, Fisher B, et al. Paclitaxel after doxorubicin plus cyclophosphamide as adjuvant chemotherapy for nodepositive breast cancer: results from NSABP B-28. J Clin Oncol. 2005;23(16):3686-96.

10. Fayers PM, Aaronson NK, Bjordal K, Groenvold M, Curran D, Bottomley A. On behalf of the EORTC quality of life group. The EORTC QLQ-C30 Scoring Manual. 3rd Edition. Brussels: European Organisation for Research and Treatment of Cancer; 2001.

11. Osoba D. Rationale for the timing of health-related qualityof-life (HQL) assessments in oncological palliative therapy. Cancer Treat Rev. 1996;22 Suppl A:69-73.

12. Kenney LB, Yasui Y, Inskip PD, Hammond S, Neglia JP, Mertens AC, et al. Breast cancer after childhood cancer: a report from the childhood cancer survivor study. Ann Intern Med. 2004;141(8):590-7.

13. Friedman DL, Rovo A, Leisenring W, Locasciulli A, Flowers ME, Tichelli A, et al. Increased risk of breast cancer among survivors of allogeneic hematopoietic cell transplantation: a report from the FHCRC and the EBMTlate effect working party. Blood. 2008;111(2):939-44.

14. Smith-Warner SA, Spiegelman D, Yaun SS, van den Brandt PA, Folsom AR, Goldbohm RA, et al. Alcohol and breast cancer in women: a pooled analysis of cohort studies. JAMA. 1998;279(7):535-40.

15. Hamajima $N$, Hirose $K$, Tajima $K$, Rohan $T$, Calle EE, Heath CW Jr, et al. Alcohol, tobacco and breast cancer - collaborative reanalysis of individual data from 53 epidemiological studies, including 58,515 women with breast cancer and 95,067 women without the disease. Br J Cancer. 2002;87(11):1234-45.

16. Degnim AC, Visscher DW, Berman HK, Frost MH, Sellers TA, Vierkant RA, et al. Stratification of breast cancer risk in women with atypia: a mayo cohort study. J Clin Oncol. 2007;25(19):2671-7.

17. Brunton LL, Lazo JS, Parker KL. Antineoplastic agents. In: Goodman and Gilman's The Pharmacological Basis of Therapeutics. 11th Edition. New York, NY: McGraw-Hill; 2006.

18. Bottomley A, Therasse P, Piccart M, Efficace F, Coens C, Gotay $\mathrm{C}$, et al. Health-related quality of life in survivors of locally advanced breast cancer: an International Randomised Controlled Phase III Trial. Lancet Oncol. 2005;6(5):287-94.

19. Martín M, Lluch A, Seguí MA, Ruiz A, Ramos M, Adrover E, et al. Toxicity and health-related quality of life in breast cancer patients receiving adjuvant docetaxel, doxorubicin, cyclophosphamide (TAC) or 5-fluorouracil, doxorubicin and cyclophosphamide (FAC): impact of adding primary prophylactic granulocyte-colony stimulating factor to the TAC regimen. Ann Oncol. 2006;17(8):1205-12.

Cite this article as: Chauhan N, Haridas K, Poddar I, Prasad K, Alwar MC, Kasturi DP. Comparative study of quality of life in breast cancer patients receiving two different chemotherapy regimens using European Organization for Research and Treatment of Cancer Quality of Questionnaire-Core 30 questionnaire module; for tolerability and safety. Int J Basic Clin Pharmacol 2015;4:664-72. 